\title{
A SHARP GENERAL OSTROWSKI TYPE INEQUALITY
}

\section{ZHENG LIU}

(Received 5 November 2009)

\section{Abstract}

A new sharp general Ostrowski type inequality in $L_{\infty}$ norm is established. Some special cases are discussed.

2010 Mathematics subject classification: primary 26D15.

Keywords and phrases: sharp inequality, Ostrowski type inequality, midpoint type inequality, trapezoid type inequality, Simpson type inequality.

\section{Introduction}

In 1998, Cerone et al. [1] proved the following Ostrowski type inequality in $L_{\infty}$ norm.

THEOREM 1.1. Let $f:[a, b] \rightarrow \mathbf{R}$ be such that $f^{(n-1)}(n \geq 1)$ is absolutely continuous on $[a, b]$ and $f^{(n)} \in L_{\infty}[a, b]$. Then for all $x \in[a, b]$,

$$
\begin{aligned}
& \left|\int_{a}^{b} f(t) d t-\sum_{k=0}^{n-1}\left[\frac{(b-x)^{k+1}+(-1)^{k}(x-a)^{k+1}}{(k+1) !}\right] f^{(k)}(x)\right| \\
& \quad \leq \frac{\left\|f^{(n)}\right\|_{\infty}}{(n+1) !}\left[(x-a)^{n+1}+(b-x)^{n+1}\right],
\end{aligned}
$$

where

$$
\left\|f^{(n)}\right\|_{\infty}:=\underset{x \in[a, b]}{\operatorname{ess} \sup }\left|f^{(n)}(x)\right|
$$

is the usual Lebesgue norm on $L_{\infty}[a, b]$.

In 2008, the author [3] proved the following Ostrowski type inequality in $L_{\infty}$ norm.

(C) 2010 Australian Mathematical Publishing Association Inc. 0004-9727/2010 \$16.00 
THEOREM 1.2. Let $f:[a, b] \rightarrow \mathbf{R}$ be such that $f^{\prime}$ is absolutely continuous on $[a, b]$ and $f^{\prime \prime} \in L_{\infty}[a, b]$. Then for all $x \in[a, b]$,

$$
\begin{aligned}
& \mid \int_{a}^{b} f(t) d t-(b-a)\left[(1-\theta) f(x)+\theta \frac{f(a)+f(b)}{2}\right. \\
& \left.\quad-(1-\theta)\left(x-\frac{a+b}{2}\right) f^{\prime}(x)\right] \mid \\
& \quad \leq\left\|f^{\prime \prime}\right\|_{\infty} I(\theta, x),
\end{aligned}
$$

where

$$
I(\theta, x)=\left\{\begin{array}{l}
\left(\frac{a+b}{2}-x\right)\left[\frac{1}{3}\left(x-\frac{a+b}{2}\right)^{2}+\left(\frac{1}{4}-\frac{\theta}{2}\right)(b-a)^{2}\right] \\
\quad+\frac{\theta^{3}(b-a)^{3}}{6}, \quad \text { for } a \leq x \leq a+\theta(b-a), \\
\frac{1-\theta}{2}(b-a)\left(x-\frac{a+b}{2}\right)^{2}+\left(\frac{\theta^{3}}{3}-\frac{\theta}{8}+\frac{1}{24}\right) \\
\times(b-a)^{3}, \quad \text { for } a+\theta(b-a)<x<b-\theta(b-a), \\
\left(x-\frac{a+b}{2}\right)\left[\frac{1}{3}\left(x-\frac{a+b}{2}\right)^{2}+\left(\frac{1}{4}-\frac{\theta}{2}\right)(b-a)^{2}\right] \\
+\frac{\theta^{3}(b-a)^{3}}{6}, \quad \text { for } b-\theta(b-a) \leq x \leq b,
\end{array}\right.
$$

for $0 \leq \theta<\frac{1}{2}$, and

$$
I(\theta, x)=\left\{\begin{array}{l}
\left(\frac{a+b}{2}-x\right)\left[\frac{1}{3}\left(x-\frac{a+b}{2}\right)^{2}+\left(\frac{1}{4}-\frac{\theta}{2}\right)(b-a)^{2}\right] \\
\quad+\frac{\theta^{3}(b-a)^{3}}{6}, \quad \text { for } a \leq x \leq b-\theta(b-a), \\
\left(\frac{\theta}{8}-\frac{1}{24}\right)(b-a)^{3}-\frac{1-\theta}{2}(b-a) \\
\quad \times\left(x-\frac{a+b}{2}\right)^{2}, \quad \text { for } b-\theta(b-a)<x<a+\theta(b-a), \\
\left(x-\frac{a+b}{2}\right)\left[\frac{1}{3}\left(x-\frac{a+b}{2}\right)^{2}+\left(\frac{1}{4}-\frac{\theta}{2}\right)(b-a)^{2}\right] \\
+\frac{\theta^{3}(b-a)^{3}}{6}, \quad \text { for } a+\theta(b-a) \leq x \leq b,
\end{array}\right.
$$

for $\frac{1}{2} \leq \theta \leq 1$. The inequality (1.2) with (1.3) and (1.4) is sharp. 
The purpose of this paper is to derive further generalizations of the above inequalities which will also lead to some interesting special cases. We need the following result given by Pearce $e t$ al. in [4].

LEMma 1.3. Let $\left\{P_{n}\right\}_{n \in \mathbf{N}}$ and $\left\{Q_{n}\right\}_{n \in \mathbf{N}}$ be two sequences of harmonic polynomials, that is,

$$
P_{n}^{\prime}(t)=P_{n-1}(t), \quad P_{0}(t)=1, \quad t \in \mathbf{R},
$$

and

$$
Q_{n}^{\prime}(t)=Q_{n-1}(t), \quad Q_{0}(t)=1, \quad t \in \mathbf{R} .
$$

Set

$$
S_{n}(t, x):= \begin{cases}P_{n}(t), & t \in[a, x], \\ Q_{n}(t), & t \in(x, b] .\end{cases}
$$

Then we have the identity

$$
\begin{gathered}
\int_{a}^{b} f(t) d t=\sum_{k=1}^{n}(-1)^{k+1}\left[Q_{k}(b) f^{(k-1)}(b)+\left(P_{k}(x)-Q_{k}(x)\right) f^{(k-1)}(x)\right. \\
\left.-P_{k}(a) f^{(k-1)}(a)\right]+(-1)^{n} \int_{a}^{b} S_{n}(t, x) f^{(n)}(t) d t
\end{gathered}
$$

provided that $f:[a, b] \rightarrow \mathbf{R}$ is such that $f^{(n-1)}$ is absolutely continuous on $[a, b]$.

\section{The results}

LeMma 2.1. Let $f:[a, b] \rightarrow \mathbf{R}$ be such that $f^{(n-1)}(n \geq 1)$ is absolutely continuous on $[a, b]$ and $f^{(n)} \in L_{\infty}[a, b]$. Then for all $x \in[a, b]$ and any $\theta \in[0,1]$ we have the identity

$$
\begin{aligned}
\int_{a}^{b} f(t) d t= & \frac{b-a}{2}[\theta f(a)+2(1-\theta) f(x)+\theta f(b)] \\
& +\sum_{k=1}^{n-1}\left\{\frac{(-1)^{k}(x-a)^{k+1}+(b-x)^{k+1}}{(k+1) !}\right. \\
& \left.-\frac{\theta(b-a)\left[(-1)^{k}(x-a)^{k}+(b-x)^{k}\right]}{2 k !}\right\} f^{(k)}(x) \\
& +(-1)^{n} \int_{a}^{b} K_{n}(t, x, \theta) f^{(n)}(t) d t
\end{aligned}
$$


where

$$
K_{n}(t, x, \theta):= \begin{cases}\frac{(t-a)^{n}}{n !}-\frac{\theta(b-a)(t-a)^{n-1}}{2(n-1) !}, & t \in[a, x], \\ \frac{(t-b)^{n}}{n !}+\frac{\theta(b-a)(t-b)^{n-1}}{2(n-1) !}, & t \in(x, b] .\end{cases}
$$

PROOF. The proof is immediate from identity (1.5) in Lemma 1.3.

THEOREM 2.2. Let $f:[a, b] \rightarrow \mathbf{R}$ be such that $f^{(n-1)}(n \geq 1)$ is absolutely continuous on $[a, b]$ and $f^{(n)} \in L_{\infty}[a, b]$. Then for all $x \in[a, b]$ and any $\theta \in[0,1]$,

$$
\begin{aligned}
& \mid \int_{a}^{b} f(t) d t-\frac{b-a}{2}[\theta f(a)+2(1-\theta) f(x)+\theta f(b)] \\
& \quad-\sum_{k=1}^{n-1}\left\{\frac{(-1)^{k}(x-a)^{k+1}+(b-x)^{k+1}}{(k+1) !}\right. \\
& \left.\quad-\frac{\theta(b-a)\left[(-1)^{k}(x-a)^{k}+(b-x)^{k}\right]}{2 k !}\right\} f^{(k)}(x) \mid \\
& \quad \leq I_{n}(\theta, x)\left\|f^{(n)}\right\|_{\infty},
\end{aligned}
$$

where

$$
I_{n}(\theta, x)=\left\{\begin{array}{l}
\frac{(b-x)^{n+1}-(x-a)^{n+1}}{(n+1) !}-\frac{\theta(b-a)\left[(b-x)^{n}-(x-a)^{n}\right]}{2 n !} \\
+\frac{n^{n} \theta^{n+1}(b-a)^{n+1}}{(n+1) ! 2^{n}}, \quad \text { for } a \leq x \leq a+\frac{n \theta}{2}(b-a), \\
\frac{(x-a)^{n+1}+(b-x)^{n+1}}{(n+1) !}-\frac{\theta(b-a)\left[(x-a)^{n}+(b-x)^{n}\right]}{2 n !} \\
+\frac{n^{n} \theta^{n+1}(b-a)^{n+1}}{(n+1) ! 2^{n-1}}, \\
\frac{(x-a)^{n+1}-(b-x)^{n+1}}{(n+1) !}-\frac{\theta(b-a)\left[(x-a)^{n}-(b-x)^{n}\right]}{2 n !} \\
+\frac{n^{n} \theta^{n+1}(b-a)^{n+1}}{(n+1) ! 2^{n}}, \quad \text { for } b-\frac{n \theta}{2}(b-a) \leq x \leq b,
\end{array}\right.
$$


for $0 \leq n \theta<1$, and

$$
I_{n}(\theta, x)=\left\{\begin{array}{l}
\frac{(b-x)^{n+1}-(x-a)^{n+1}}{(n+1) !}-\frac{\theta(b-a)\left[(b-x)^{n}-(x-a)^{n}\right]}{2 n !} \\
+\frac{n^{n} \theta^{n+1}(b-a)^{n+1}}{(n+1) ! 2^{n}}, \quad \text { for } a \leq x \leq b-\frac{n \theta}{2}(b-a), \\
-\frac{(x-a)^{n+1}+(b-x)^{n+1}}{(n+1) !}+\frac{\theta(b-a)\left[(x-a)^{n}+(b-x)^{n}\right]}{2 n !}, \\
\text { for } b-\frac{n \theta}{2}(b-a)<x<a+\frac{n \theta}{2}(b-a), \\
\frac{(x-a)^{n+1}-(b-x)^{n+1}}{(n+1) !}-\frac{\theta(b-a)\left[(x-a)^{n}-(b-x)^{n}\right]}{2 n !} \\
+\frac{n^{n} \theta^{n+1}(b-a)^{n+1}}{(n+1) ! 2^{n}}, \quad \text { for } a+\frac{n \theta}{2}(b-a) \leq x \leq b,
\end{array}\right.
$$

for $1 \leq n \theta<2$, and

$$
I_{n}(\theta, x)=-\frac{(x-a)^{n+1}+(b-x)^{n+1}}{(n+1) !}+\frac{\theta(b-a)\left[(x-a)^{n}+(b-x)^{n}\right]}{2 n !}
$$

for $n \theta \geq 2$. Inequality (2.3) with (2.4), (2.5) and (2.6) is sharp.

PROOF. Using identity (2.1) in Lemma 2.1, we get

$$
\begin{aligned}
& \mid \int_{a}^{b} f(t) d t-\frac{b-a}{2}[\theta f(a)+2(1-\theta) f(x)+\theta f(b)] \\
& \quad-\sum_{k=1}^{n-1}\left\{\frac{(-1)^{k}(x-a)^{k+1}+(b-x)^{k+1}}{(k+1) !}\right. \\
& \left.\quad-\frac{\theta(b-a)\left[(-1)^{k}(x-a)^{k}+(b-x)^{k}\right]}{2 k !}\right\} f^{(k)}(x) \mid \\
& \quad \leq I_{n}(\theta, x)\left\|f^{(n)}\right\|_{\infty},
\end{aligned}
$$

where

$$
I_{n}(\theta, x)=\int_{a}^{b}\left|K_{n}(t, x, \theta)\right| d t
$$

Then by (2.2),

$$
\begin{aligned}
n ! I_{n}(\theta, x)= & \int_{a}^{x}\left|(t-a)^{n}-\frac{n \theta}{2}(b-a)(t-a)^{n-1}\right| d t \\
& +\int_{x}^{b}\left|(t-b)^{n}+\frac{n \theta}{2}(b-a)(t-b)^{n-1}\right| d t .
\end{aligned}
$$


The last two integrals in (2.8) can be calculated as follows. For brevity, we put

$$
\begin{array}{ll}
P(t):=(t-a)^{n-1}\left[t-a-\frac{n \theta}{2}(b-a)\right], & t \in[a, b], \\
Q(t):=(t-b)^{n-1}\left[t-b+\frac{n \theta}{2}(b-a)\right], & t \in[a, b],
\end{array}
$$

where $\theta \in[0,1]$. It is clear that both $P(t)$ and $Q(t)$ have only one zero in $(a, b)$ for $0<n \theta<2$. Let $t_{1}=a+n \theta(b-a) / 2$ and $t_{2}=b-n \theta(b-a) / 2$. It is easy to see that $a \leq t_{1}<(a+b) / 2<t_{2} \leq b$ if and only if $0 \leq n \theta<1$ as well as $a \leq t_{2} \leq(a+b) / 2 \leq$ $t_{1} \leq b$ if and only if $1 \leq n \theta \leq 2$. Thus we have: for an odd $n$ with $0 \leq n \theta<1$,

$$
\begin{aligned}
n ! I_{n}(\theta, x)=- & \int_{a}^{x}\left[(t-a)^{n}-\frac{n \theta}{2}(b-a)(t-a)^{n-1}\right] d t \\
& -\int_{x}^{t_{2}}\left[(t-b)^{n}+\frac{n \theta}{2}(b-a)(t-b)^{n-1}\right] d t \\
& +\int_{t_{2}}^{b}\left[(t-b)^{n}+\frac{n \theta}{2}(b-a)(t-b)^{n-1}\right] d t \\
= & \frac{(b-x)^{n+1}-(x-a)^{n+1}}{n+1}-\frac{\theta(b-a)\left[(b-x)^{n}-(x-a)^{n}\right]}{2} \\
& +\frac{n^{n} \theta^{n+1}(b-a)^{n+1}}{(n+1) 2^{n}}
\end{aligned}
$$

when $a \leq x \leq a+n \theta(b-a) / 2$,

$$
\begin{aligned}
n ! I_{n}(\theta, x)=- & \int_{a}^{t_{1}}\left[(t-a)^{n}-\frac{n \theta}{2}(b-a)(t-a)^{n-1}\right] d t \\
& +\int_{t_{1}}^{x}\left[(t-a)^{n}-\frac{n \theta}{2}(b-a)(t-a)^{n-1}\right] d t \\
& -\int_{x}^{t_{2}}\left[(t-b)^{n}+\frac{n \theta}{2}(b-a)(t-b)^{n-1}\right] d t \\
& +\int_{t_{2}}^{b}\left[(t-b)^{n}+\frac{n \theta}{2}(b-a)(t-b)^{n-1}\right] d t \\
= & \frac{(x-a)^{n+1}+(b-x)^{n+1}}{n+1}-\frac{\theta(b-a)\left[(x-a)^{n}+(b-x)^{n}\right]}{2} \\
& +\frac{n^{n} \theta^{n+1}(b-a)^{n+1}}{(n+1) 2^{n-1}}
\end{aligned}
$$


when $a+n \theta(b-a) / 2<x<b-n \theta(b-a) / 2$,

$$
\begin{aligned}
n ! I_{n}(\theta, x)=- & \int_{a}^{t_{1}}\left[(t-a)^{n}-\frac{n \theta}{2}(b-a)(t-a)^{n-1}\right] d t \\
& +\int_{t_{1}}^{x}\left[(t-a)^{n}-\frac{n \theta}{2}(b-a)(t-a)^{n-1}\right] d t \\
& +\int_{x}^{b}\left[(t-b)^{n}+\frac{n \theta}{2}(b-a)(t-b)^{n-1}\right] d t \\
= & \frac{(x-a)^{n+1}-(b-x)^{n+1}}{n+1}-\frac{\theta(b-a)\left[(x-a)^{n}-(b-x)^{n}\right]}{2} \\
& +\frac{n^{n} \theta^{n+1}(b-a)^{n+1}}{(n+1) 2^{n}}
\end{aligned}
$$

when $b-n \theta(b-a) / 2 \leq x \leq b$; for an even $n$ with $0 \leq n \theta<1$,

$$
\begin{aligned}
n ! I_{n}(\theta, x)=- & \int_{a}^{x}\left[(t-a)^{n}-\frac{n \theta}{2}(b-a)(t-a)^{n-1}\right] d t \\
& +\int_{x}^{t_{2}}\left[(t-b)^{n}+\frac{n \theta}{2}(b-a)(t-b)^{n-1}\right] d t \\
& \quad-\int_{t_{2}}^{b}\left[(t-b)^{n}+\frac{n \theta}{2}(b-a)(t-b)^{n-1}\right] d t \\
= & \frac{(b-x)^{n+1}-(x-a)^{n+1}}{n+1}-\frac{\theta(b-a)\left[(b-x)^{n}-(x-a)^{n}\right]}{2} \\
& +\frac{n^{n} \theta^{n+1}(b-a)^{n+1}}{(n+1) 2^{n}}
\end{aligned}
$$

when $a \leq x \leq a+n \theta(b-a) / 2$,

$$
\begin{aligned}
n ! I_{n}(\theta, x)=- & \int_{a}^{t_{1}}\left[(t-a)^{n}-\frac{n \theta}{2}(b-a)(t-a)^{n-1}\right] d t \\
& +\int_{t_{1}}^{x}\left[(t-a)^{n}-\frac{n \theta}{2}(b-a)(t-a)^{n-1}\right] d t \\
& +\int_{x}^{t_{2}}\left[(t-b)^{n}+\frac{n \theta}{2}(b-a)(t-b)^{n-1}\right] d t \\
& -\int_{t_{2}}^{b}\left[(t-b)^{n}+\frac{n \theta}{2}(b-a)(t-b)^{n-1}\right] d t \\
= & \frac{(x-a)^{n+1}+(b-x)^{n+1}}{n+1}-\frac{\theta(b-a)\left[(x-a)^{n}+(b-x)^{n}\right]}{2} \\
& +\frac{n^{n} \theta^{n+1}(b-a)^{n+1}}{(n+1) 2^{n-1}}
\end{aligned}
$$


when $a+n \theta(b-a) / 2<x<b-n \theta(b-a) / 2$,

$$
\begin{aligned}
n ! I_{n}(\theta, x)=- & \int_{a}^{t_{1}}\left[(t-a)^{n}-\frac{n \theta}{2}(b-a)(t-a)^{n-1}\right] d t \\
& +\int_{t_{1}}^{x}\left[(t-a)^{n}-\frac{n \theta}{2}(b-a)(t-a)^{n-1}\right] d t \\
& \quad-\int_{x}^{b}\left[(t-b)^{n}+\frac{n \theta}{2}(b-a)(t-b)^{n-1}\right] d t \\
= & \frac{(x-a)^{n+1}-(b-x)^{n+1}}{n+1}-\frac{\theta(b-a)\left[(x-a)^{n}-(b-x)^{n}\right]}{2} \\
& +\frac{n^{n} \theta^{n+1}(b-a)^{n+1}}{(n+1) 2^{n}}
\end{aligned}
$$

when $b-n \theta(b-a) / 2 \leq x \leq b$; for an odd $n$ with $1 \leq n \theta<2$,

$$
\begin{aligned}
n ! I_{n}(\theta, x)=- & \int_{a}^{x}\left[(t-a)^{n}-\frac{n \theta}{2}(b-a)(t-a)^{n-1}\right] d t \\
& -\int_{x}^{t_{2}}\left[(t-b)^{n}+\frac{n \theta}{2}(b-a)(t-b)^{n-1}\right] d t \\
& +\int_{t_{2}}^{b}\left[(t-b)^{n}+\frac{n \theta}{2}(b-a)(t-b)^{n-1}\right] d t \\
= & \frac{(b-x)^{n+1}-(x-a)^{n+1}}{n+1}-\frac{\theta(b-a)\left[(b-x)^{n}-(x-a)^{n}\right]}{2} \\
& +\frac{n^{n} \theta^{n+1}(b-a)^{n+1}}{(n+1) 2^{n}}
\end{aligned}
$$

when $a \leq x \leq b-n \theta(b-a) / 2$,

$$
\begin{aligned}
n ! I_{n}(\theta, x)=- & \int_{a}^{x}\left[(t-a)^{n}-\frac{n \theta}{2}(b-a)(t-a)^{n-1}\right] d t \\
& \quad+\int_{x}^{b}\left[(t-b)^{n}+\frac{n \theta}{2}(b-a)(t-b)^{n-1}\right] d t \\
= & -\frac{(x-a)^{n+1}+(b-x)^{n+1}}{n+1}+\frac{\theta(b-a)\left[(x-a)^{n}+(b-x)^{n}\right]}{2}
\end{aligned}
$$


when $b-n \theta(b-a) / 2<x<a+n \theta(b-a) / 2$,

$$
\begin{aligned}
n ! I_{n}(\theta, x)=- & \int_{a}^{t_{1}}\left[(t-a)^{n}-\frac{n \theta}{2}(b-a)(t-a)^{n-1}\right] d t \\
& +\int_{t_{1}}^{x}\left[(t-a)^{n}-\frac{n \theta}{2}(b-a)(t-a)^{n-1}\right] d t \\
& +\int_{x}^{b}\left[(t-b)^{n}+\frac{n \theta}{2}(b-a)(t-b)^{n-1}\right] d t \\
= & \frac{(x-a)^{n+1}-(b-x)^{n+1}}{n+1}-\frac{\theta(b-a)\left[(x-a)^{n}-(b-x)^{n}\right]}{2} \\
& +\frac{n^{n} \theta^{n+1}(b-a)^{n+1}}{(n+1) 2^{n}}
\end{aligned}
$$

when $a+n \theta(b-a) / 2 \leq x \leq b$; for an even $n$ with $1 \leq n \theta<2$,

$$
\begin{aligned}
n ! I_{n}(\theta, x)=- & \int_{a}^{x}\left[(t-a)^{n}-\frac{n \theta}{2}(b-a)(t-a)^{n-1}\right] d t \\
& +\int_{x}^{t_{2}}\left[(t-b)^{n}+\frac{n \theta}{2}(b-a)(t-b)^{n-1}\right] d t \\
& \quad-\int_{t_{2}}^{b}\left[(t-b)^{n}+\frac{n \theta}{2}(b-a)(t-b)^{n-1}\right] d t \\
= & \frac{(b-x)^{n+1}-(x-a)^{n+1}}{n+1}-\frac{\theta(b-a)\left[(b-x)^{n}-(x-a)^{n}\right]}{2} \\
& +\frac{n^{n} \theta^{n+1}(b-a)^{n+1}}{(n+1) 2^{n}}
\end{aligned}
$$

when $a \leq x \leq a+n \theta(b-a) / 2$,

$$
\begin{aligned}
n ! I_{n}(\theta, x)=- & \int_{a}^{x}\left[(t-a)^{n}-\frac{n \theta}{2}(b-a)(t-a)^{n-1}\right] d t \\
& \quad-\int_{x}^{b}\left[(t-b)^{n}+\frac{n \theta}{2}(b-a)(t-b)^{n-1}\right] d t \\
= & -\frac{(x-a)^{n+1}+(b-x)^{n+1}}{n+1}+\frac{\theta(b-a)\left[(x-a)^{n}+(b-x)^{n}\right]}{2}
\end{aligned}
$$


when $a+n \theta(b-a) / 2<x<b-n \theta(b-a) / 2$,

$$
\begin{aligned}
n ! I_{n}(\theta, x)=- & \int_{a}^{t_{1}}\left[(t-a)^{n}-\frac{n \theta}{2}(b-a)(t-a)^{n-1}\right] d t \\
& +\int_{t_{1}}^{x}\left[(t-a)^{n}-\frac{n \theta}{2}(b-a)(t-a)^{n-1}\right] d t \\
& \quad-\int_{x}^{b}\left[(t-b)^{n}+\frac{n \theta}{2}(b-a)(t-b)^{n-1}\right] d t \\
= & \frac{(x-a)^{n+1}-(b-x)^{n+1}}{n+1}-\frac{\theta(b-a)\left[(x-a)^{n}-(b-x)^{n}\right]}{2} \\
& +\frac{n^{n} \theta^{n+1}(b-a)^{n+1}}{(n+1) 2^{n}}
\end{aligned}
$$

when $b-n \theta(b-a) / 2 \leq x \leq b$; for an odd $n$ with $n \theta \geq 2$,

$$
\begin{aligned}
n ! I_{n}(\theta, x)=- & \int_{a}^{x}\left[(t-a)^{n}-\frac{n \theta}{2}(b-a)(t-a)^{n-1}\right] d t \\
& +\int_{x}^{b}\left[(t-b)^{n}+\frac{n \theta}{2}(b-a)(t-b)^{n-1}\right] d t \\
= & -\frac{(x-a)^{n+1}+(b-x)^{n+1}}{n+1}+\frac{\theta(b-a)\left[(x-a)^{n}+(b-x)^{n}\right]}{2}
\end{aligned}
$$

when $a \leq x \leq b$; and for an even $n$ with $n \theta \geq 2$,

$$
\begin{aligned}
n ! I_{n}(\theta, x)= & -\int_{a}^{x}\left[(t-a)^{n}-\frac{n \theta}{2}(b-a)(t-a)^{n-1}\right] d t \\
& -\int_{x}^{b}\left[(t-b)^{n}+\frac{n \theta}{2}(b-a)(t-b)^{n-1}\right] d t \\
= & -\frac{(x-a)^{n+1}+(b-x)^{n+1}}{n+1}+\frac{\theta(b-a)\left[(x-a)^{n}+(b-x)^{n}\right]}{2}
\end{aligned}
$$

when $a \leq x \leq b$. Consequently, inequality (2.3) with (2.4), (2.5) and (2.6) follows from (2.7) and (2.9)-(2.22).

We now prove that inequality (2.3) with (2.4), (2.5) and (2.6) is sharp. Indeed, we can choose $f$ to attain equality in (2.3) with (2.4), (2.5) and (2.6). If $n$ is odd, we may construct $f$ such that:

$$
f^{(n-1)}(t)= \begin{cases}-t, & a \leq t<t_{2} \\ t-2 t_{2}, & t_{2} \leq t \leq b\end{cases}
$$


when $a \leq x \leq t_{1}$,

$$
f^{(n-1)}(t)= \begin{cases}-t, & a \leq t<t_{1} \\ t-2 t_{1}, & t_{1} \leq t<x \\ -t+2\left(x-t_{1}\right), & x \leq t<t_{2} \\ t+2\left(x-t_{1}-t_{2}\right), & t_{2} \leq t \leq b\end{cases}
$$

when $t_{1}<x<t_{2}$, and

$$
f^{(n-1)}(t)= \begin{cases}-t, & a \leq t<t_{1}, \\ t-2 t_{1}, & t_{1} \leq t \leq b,\end{cases}
$$

when $t_{2} \leq x \leq b$ for $0 \leq n \theta<1$;

$$
f^{(n-1)}(t)= \begin{cases}-t, & a \leq t<t_{2}, \\ t-2 t_{2}, & t_{2} \leq t \leq b\end{cases}
$$

when $a \leq x \leq t_{2}$,

$$
f^{(n-1)}(t)= \begin{cases}-t, & a \leq t<x \\ t-2 x, & x \leq t \leq b\end{cases}
$$

when $t_{2}<x<t_{1}$,

$$
f^{(n-1)}(t)= \begin{cases}-t, & a \leq t<t_{1}, \\ t-2 t_{1}, & t_{1} \leq t \leq b,\end{cases}
$$

when $t_{1} \leq x \leq b$ for $1 \leq n \theta<2$; and

$$
f^{(n-1)}(t)= \begin{cases}-t, & a \leq t<x, \\ t-2 x, & x \leq t \leq b,\end{cases}
$$

when $a \leq x \leq b$ for $n \theta \geq 2$.

If $n$ is even, we may construct $f$ such that:

$$
f^{(n-1)}(t)= \begin{cases}-t, & a \leq t<x \\ t-2 x, & x \leq t<t_{2} \\ -t+2\left(t_{2}-x\right), & t_{2} \leq t \leq b\end{cases}
$$

when $a \leq x \leq t_{1}$,

$$
f^{(n-1)}(t)= \begin{cases}-t, & a \leq t<t_{1} \\ t-2 t_{1}, & t_{1} \leq t<t_{2} \\ -t+2\left(t_{2}-t_{1}\right), & t_{2} \leq t \leq b\end{cases}
$$


when $t_{1}<x<t_{2}$, and

$$
f^{(n-1)}(t)= \begin{cases}-t, & a \leq t<t_{1}, \\ t-2 t_{1}, & t_{1} \leq t \leq x, \\ -t+2\left(x-t_{1}\right), & x \leq t \leq b,\end{cases}
$$

when $t_{2} \leq x \leq b$ for $0 \leq n \theta<1$;

$$
f^{(n-1)}(t)= \begin{cases}-t, & a \leq t<x, \\ t-2 x, & x<t \leq t_{2}, \\ -t+2\left(t_{2}-x\right), & t_{2} \leq t \leq b,\end{cases}
$$

when $a \leq x \leq t_{2}$,

$$
f^{(n-1)}(t)=-t, \quad a \leq t \leq b,
$$

when $t_{2}<x<t_{1}$, and

$$
f^{(n-1)}(t)= \begin{cases}-t, & a \leq t<t_{1}, \\ t-2 t_{1}, & t_{1} \leq t<x, \\ -t+2\left(x-t_{1}\right), & x \leq t \leq b,\end{cases}
$$

when $t_{1} \leq x \leq b$ for $1 \leq n \theta<2$;

$$
f^{(n-1)}(t)=-t, \quad a \leq t \leq b,
$$

when $a \leq x \leq b$ for $n \theta \geq 2$.

Clearly, all the above $f^{(n-1)}$ are absolutely continuous on $[a, b]$, and then, if $n$ is odd,

$$
f^{(n)}(t)= \begin{cases}-1, & a<t<t_{2}, \\ 1, & t_{2}<t<b,\end{cases}
$$

when $a \leq x \leq t_{1}$,

$$
f^{(n)}(t)= \begin{cases}-1, & a<t<t_{1} \\ 1, & t_{1}<t<x \\ -1, & x<t<t_{2} \\ 1, & t_{2}<t<b\end{cases}
$$

when $t_{1}<x<t_{2}$, and

$$
f^{(n)}(t)= \begin{cases}-1, & a<t<t_{1}, \\ 1, & t_{1}<t<b,\end{cases}
$$

when $t_{2} \leq x \leq b$ for $0 \leq n \theta<1$;

$$
f^{(n)}(t)= \begin{cases}-1, & a<t<t_{2}, \\ 1, & t_{2}<t<b,\end{cases}
$$


when $a \leq x \leq t_{2}$,

$$
f^{(n)}(t)= \begin{cases}-1, & a<t<x \\ 1, & x<t<b\end{cases}
$$

when $t_{2}<x<t_{1}$, and

$$
f^{(n)}(t)= \begin{cases}-1, & a<t<t_{1}, \\ 1, & t_{1}<t<b,\end{cases}
$$

when $t_{1} \leq x \leq b$, for $1 \leq n \theta<2$; and

$$
f^{(n)}(t)= \begin{cases}-1, & a<t<x, \\ 1, & x<t<b\end{cases}
$$

when $a \leq x \leq b$ for $n \theta \geq 2$. If $n$ is even:

$$
f^{(n)}(t)= \begin{cases}-1, & a<t<x \\ 1, & x<t<t_{2}, \\ -1, & t_{2}<t<b\end{cases}
$$

when $a \leq x \leq t_{1}$,

$$
f^{(n)}(t)= \begin{cases}-1, & a<t<t_{1} \\ 1, & t_{1}<t<t_{2} \\ -1, & t_{2}<t<b\end{cases}
$$

when $t_{1}<x<t_{2}$, and

$$
f^{(n)}(t)= \begin{cases}-1, & a<t<t_{1}, \\ 1, & t_{1}<t<x, \\ -1, & x<t<b,\end{cases}
$$

when $t_{2} \leq x \leq b$, for $0 \leq n \theta<1$;

$$
f^{(n)}(t)= \begin{cases}-1, & a<t<x, \\ 1, & x<t<t_{2}, \\ -1, & t_{2}<t<b,\end{cases}
$$

when $a \leq x \leq t_{2}$,

$$
f^{(n)}(t)=-1, \quad a<t<b,
$$


when $t_{2}<x<t_{1}$,

$$
f^{(n)}(t)= \begin{cases}-1, & a<t<t_{1} \\ 1, & t_{1}<t<x \\ -1, & x<t<b\end{cases}
$$

when $t_{1} \leq x \leq b$, for $1 \leq n \theta<2$;

$$
f^{(n)}(t)=-1, \quad a<t<b,
$$

when $a \leq x \leq b$, for $n \theta \geq 2$, which satisfy the condition of Theorem 2.2 with $\left\|f^{(n)}\right\|_{\infty}=1$. The proof is complete.

REMARK 2.3. It is clear that Theorem 1.1 is just the special case $\theta=0$ of Theorem 2.2 without a proof on sharpness of inequality (1.1), and Theorem 1.2 is just the special case $n=2$ of Theorem 2.2.

COROllary 2.4. Let the assumptions of Theorem 2.2 hold. Then for $n=1,2$ we have sharp trapezoid inequalities

$$
\left|\int_{a}^{b} f(t) d t-\frac{b-a}{2}[f(a)+f(b)]\right| \leq \frac{(b-a)^{2}}{4}\left\|f^{\prime}\right\|_{\infty}
$$

and

$$
\left|\int_{a}^{b} f(t) d t-\frac{b-a}{2}[f(a)+f(b)]\right| \leq \frac{(b-a)^{3}}{12}\left\|f^{\prime \prime}\right\|_{\infty},
$$

and for $n \geq 3$ we have sharp trapezoid type inequalities

$$
\begin{aligned}
& \mid \int_{a}^{b} f(t) d t-\frac{b-a}{2}[f(a)+f(b)] \\
& \quad-\sum_{k=1}^{n-1}\left\{\frac{(-1)^{k}(x-a)^{k+1}+(b-x)^{k+1}}{(k+1) !}\right. \\
& \left.\quad-\frac{\theta(b-a)\left[(-1)^{k}(x-a)^{k}+(b-x)^{k}\right]}{2 k !}\right\} f^{(k)}(x) \mid \\
& \quad \leq\left\|f^{(n)}\right\|_{\infty}\left[\frac{(x-a)^{n}+(b-x)^{n}}{2 n !}(b-a)-\frac{(x-a)^{n+1}+(b-x)^{n+1}}{(n+1) !}\right] .
\end{aligned}
$$

Proof. Letting $\theta=1$ in (2.3) with (2.4), (2.5) and (2.6) readily produces the results (2.23)-(2.25). 
COROllary 2.5. Let the assumptions of Theorem 2.2 hold. Then for $n=1,2,3$, 4, 5, we have sharp Simpson type inequalities

$$
\begin{aligned}
& \left|\int_{a}^{b} f(t) d t-\frac{b-a}{6}[f(a)+4 f(x)+f(b)]\right| \\
& \leq\left\|f^{\prime}\right\|_{\infty} \times\left\{\begin{array}{l}
\frac{2}{3}(b-a)\left(\frac{a+b}{2}-x\right)+\frac{(b-a)^{2}}{36}, \\
\quad \text { for } a \leq x \leq a+\frac{5 a+b}{6}, \\
\left.\quad \text { for } \frac{5 a+b}{6}<x<\frac{a+b}{6}\right)^{2}+\frac{5(b-a)^{2}}{36}, \\
\frac{2}{3}(b-a)\left(x-\frac{a+b}{2}\right)+\frac{(b-a)^{2}}{36}, \\
\text { for } \frac{a+5 b}{6} \leq x \leq b,
\end{array}\right. \\
& \left|\int_{a}^{b} f(t) d t-\frac{b-a}{6}[f(a)+4 f(x)+f(b)]+\frac{2(b-a)}{3}\left(x-\frac{a+b}{2}\right) f^{\prime}(x)\right| \\
& \leq\left\|f^{\prime \prime}\right\|_{\infty} \times\left\{\begin{array}{l}
\frac{1}{3}\left(\frac{a+b}{2}-x\right)^{3}+\frac{(b-a)^{2}}{12}\left(\frac{a+b}{2}-x\right)+\frac{(b-a)^{3}}{162} \\
\quad \text { for } a \leq x \leq a+\frac{2 a+b}{3} \\
\frac{b-a}{3}\left(x-\frac{a+b}{2}\right)^{2}+\frac{(b-a)^{3}}{81} \\
\quad \text { for } \frac{2 a+b}{3}<x<\frac{a+2 b}{3}, \\
\frac{1}{3}\left(x-\frac{a+b}{2}\right)^{3}+\frac{(b-a)^{2}}{12}\left(x-\frac{a+b}{2}\right)+\frac{(b-a)^{3}}{162} \\
\quad \text { for } \frac{a+2 b}{3} \leq x \leq b,
\end{array}\right.
\end{aligned}
$$




$$
\begin{aligned}
& \mid \int_{a}^{b} f(t) d t-\frac{b-a}{6}[f(a)+4 f(x)+f(b)] \\
& \quad+\frac{2(b-a)}{3}\left(x-\frac{a+b}{2}\right) f^{\prime}(x)-\frac{b-a}{3}\left(x-\frac{a+b}{2}\right)^{2} f^{\prime \prime}(x) \mid \\
& \quad \leq\left\|f^{\prime \prime \prime \prime}\right\|_{\infty} \times\left\{\begin{array}{l}
\frac{b-a}{9}\left(\frac{a+b}{2}-x\right)^{3}+\frac{(b-a)^{4}}{576}, \quad \text { for } a \leq x \leq \frac{a+b}{2}, \\
\frac{b-a}{9}\left(x-\frac{a+b}{2}\right)^{3}+\frac{(b-a)^{4}}{576}, \text { for } \frac{a+b}{2} \leq x \leq b,
\end{array}\right.
\end{aligned}
$$

$$
\begin{aligned}
& \mid \int_{a}^{b} f(t) d t-\frac{b-a}{6}[f(a)+4 f(x)+f(b)]+\frac{2(b-a)}{3}\left(x-\frac{a+b}{2}\right) f^{\prime}(x) \\
& -\frac{b-a}{3}\left(x-\frac{a+b}{2}\right)^{2} f^{\prime \prime}(x)+\frac{b-a}{9}\left(x-\frac{a+b}{2}\right)^{3} f^{\prime \prime \prime}(x) \mid \\
& \leq\left\|f^{(4)}\right\|_{\infty} \times\left\{\begin{array}{l}
\frac{1}{60}\left(\frac{a+b}{2}-x\right)^{5}+\frac{(b-a)^{2}}{72}\left(\frac{a+b}{2}-x\right)^{3} \\
\quad-\frac{(b-a)^{4}}{576}\left(\frac{a+b}{2}-x\right)+\frac{2(b-a)^{5}}{3645} \\
\text { for } a \leq x \leq a+\frac{2 a+b}{3}, \\
f(b-a)^{5}-\frac{b-a}{36}\left(x-\frac{a+b}{2}\right)^{4}, \\
1 \\
\frac{1}{60}\left(x-\frac{a+b}{2}\right)^{5}+\frac{(b-a)^{2}}{72}\left(x-\frac{a+b}{2}\right)^{3} \\
-\frac{(b-a)^{4}}{576}\left(x-\frac{a+b}{2}\right)+\frac{2(b-a)^{5}}{3645} \\
\text { for } \frac{a+2 b}{3} \leq x \leq b,
\end{array}\right.
\end{aligned}
$$


and

$$
\begin{aligned}
& \mid \int_{a}^{b} f(t) d t-\frac{b-a}{6}[f(a)+4 f(x)+f(b)] \\
& +\frac{2(b-a)}{3}\left(x-\frac{a+b}{2}\right) f^{\prime}(x)-\frac{b-a}{3}\left(x-\frac{a+b}{2}\right)^{2} f^{\prime \prime}(x) \\
& +\frac{b-a}{9}\left(x-\frac{a+b}{2}\right)^{3} f^{\prime \prime \prime}(x) \\
& +\left[\frac{(b-a)^{5}}{2880}-\frac{b-a}{36}\left(x-\frac{a+b}{2}\right)^{4}\right] f^{(4)}(x) \mid \\
& \left(\frac{b-a}{180}\left(\frac{a+b}{2}-x\right)^{5}-\frac{(b-a)^{5}}{2880}\left(\frac{a+b}{2}-x\right)\right. \\
& +\frac{625(b-a)^{6}}{3359232}, \quad \text { for } a \leq x \leq \frac{5 a+b}{6} \text {, } \\
& -\frac{1}{360}\left(x-\frac{a+b}{2}\right)^{6}-\frac{(b-a)^{2}}{288}\left(x-\frac{a+b}{2}\right)^{4} \\
& \leq\left\|f^{(5)}\right\|_{\infty} \times\left\{+\frac{(b-a)^{4}}{1152}\left(x-\frac{a+b}{2}\right)^{2}+\frac{(b-a)^{6}}{23040},\right. \\
& \text { for } \frac{5 a+b}{6}<x<\frac{a+5 b}{6} \text {, } \\
& \frac{b-a}{180}\left(x-\frac{a+b}{2}\right)^{5}-\frac{(b-a)^{5}}{2880}\left(x-\frac{a+b}{2}\right) \\
& +\frac{625(b-a)^{6}}{3359232}, \quad \text { for } \frac{a+5 b}{6} \leq x \leq b,
\end{aligned}
$$

and for $n \geq 6$ we have the sharp Simpson type inequality

$$
\begin{aligned}
& \mid \int_{a}^{b} f(t) d t-\frac{b-a}{6}[f(a)+4 f(x)+f(b)] \\
& \quad-\sum_{k=1}^{n-1}\left\{\frac{(-1)^{k}(x-a)^{k+1}+(b-x)^{k+1}}{(k+1) !}\right. \\
& \left.\quad-\frac{\theta(b-a)\left[(-1)^{k}(x-a)^{k}+(b-x)^{k}\right]}{6 k !}\right\} f^{(k)}(x) \mid \\
& \quad \leq\left\|f^{(n)}\right\|_{\infty}\left[\frac{(x-a)^{n}+(b-x)^{n}}{6 n !}(b-a)-\frac{(x-a)^{n+1}+(b-x)^{n+1}}{(n+1) !}\right] .
\end{aligned}
$$


Proof. Putting $\theta=\frac{1}{3}$ in (2.3) with (2.4), (2.5) and (2.6) readily produces the results (2.26)-(2.31).

COROLLARY 2.6. Let the assumptions of Theorem 2.2 hold. Then for $n=1,2,3$ we have sharp averaged midpoint-trapezoid type inequalities

$$
\begin{aligned}
& \left|\int_{a}^{b} f(t) d t-\frac{b-a}{4}[f(a)+2 f(x)+f(b)]\right| \\
& \left(\frac{1}{2}(b-a)\left(\frac{a+b}{2}-x\right)+\frac{(b-a)^{2}}{16}\right. \\
& \text { for } a \leq x \leq a+\frac{3 a+b}{4}, \\
& \leq\left\|f^{\prime}\right\|_{\infty} \times\left\{\begin{array}{r}
\left(x-\frac{a+b}{2}\right)^{2}+\frac{(b-a)^{2}}{8}, \\
\text { for } \frac{3 a+b}{4}<x<\frac{a+3 b}{4},
\end{array}\right. \\
& \left\{\begin{array}{l}
\frac{1}{2}(b-a)\left(x-\frac{a+b}{2}\right)+\frac{(b-a)^{2}}{16}, \\
\text { for } \frac{a+3 b}{4} \leq x \leq b,
\end{array}\right. \\
& \left|\int_{a}^{b} f(t) d t-\frac{b-a}{4}[f(a)+2 f(x)+f(b)]+\frac{b-a}{2}\left(x-\frac{a+b}{2}\right) f^{\prime}(x)\right| \\
& \leq\left\|f^{\prime \prime}\right\|_{\infty} \times \begin{cases}\frac{1}{3}\left(\frac{a+b}{2}-x\right)^{3}+\frac{(b-a)^{3}}{48}, & \text { for } a \leq x \leq \frac{a+b}{2}, \\
\frac{1}{3}\left(x-\frac{a+b}{2}\right)^{3}+\frac{(b-a)^{3}}{48}, & \text { for } \frac{a+b}{2} \leq x \leq b,\end{cases}
\end{aligned}
$$

and

$$
\begin{aligned}
& \mid \int_{a}^{b} f(t) d t-\frac{b-a}{4}[f(a)+2 f(x)+f(b)] \\
& \quad+\frac{b-a}{2}\left(x-\frac{a+b}{2}\right) f^{\prime}(x)+\left[\frac{(b-a)^{3}}{48}-\frac{b-a}{4}\left(x-\frac{a+b}{2}\right)^{2}\right] f^{\prime \prime}(x) \mid
\end{aligned}
$$




$$
\leq\left\|f^{\prime \prime \prime}\right\|_{\infty} \times\left\{\begin{array}{l}
\frac{b-a}{12}\left(\frac{a+b}{2}-x\right)^{3}-\frac{(b-a)^{3}}{48}\left(\frac{a+b}{2}-x\right) \\
\quad+\frac{9(b-a)^{4}}{1024}, \quad \text { for } a \leq x \leq a+\frac{3 a+b}{4}, \\
-\frac{1}{12}\left(x-\frac{a+b}{2}\right)^{4}+\frac{(b-a)^{4}}{192}, \\
\text { for } \frac{3 a+b}{4}<x<\frac{a+3 b}{4}, \\
\frac{b-a}{12}\left(x-\frac{a+b}{2}\right)^{3}-\frac{(b-a)^{3}}{48}\left(x-\frac{a+b}{2}\right) \\
+\frac{9(b-a)^{4}}{1024}, \quad \text { for } \frac{a+3 b}{4} \leq x \leq b,
\end{array}\right.
$$

and for $n \geq 4$ we have the sharp averaged midpoint-trapezoid type inequality

$$
\begin{aligned}
& \mid \int_{a}^{b} f(t) d t-\frac{b-a}{4}[f(a)+2 f(x)+f(b)] \\
& \quad-\sum_{k=1}^{n-1}\left\{\frac{(-1)^{k}(x-a)^{k+1}+(b-x)^{k+1}}{(k+1) !}\right. \\
& \left.\quad-\frac{\theta(b-a)\left[(-1)^{k}(x-a)^{k}+(b-x)^{k}\right]}{4 k !}\right\} f^{(k)}(x) \mid \\
& \quad \leq\left\|f^{(n)}\right\|_{\infty}\left[\frac{(x-a)^{n}+(b-x)^{n}}{4 n !}(b-a)-\frac{(x-a)^{n+1}+(b-x)^{n+1}}{(n+1) !}\right] .
\end{aligned}
$$

Proof. Putting $\theta=\frac{1}{2}$ in (2.3) with (2.4), (2.5) and (2.6) readily produces the results (2.32)-(2.35).

COROLlaRY 2.7. Let the assumptions of Theorem 2.2 hold. Then for any $\theta \in[0,1]$,

$$
\begin{aligned}
& \mid \int_{a}^{b} f(x) d x-\frac{b-a}{2}\left[\theta f(a)+2(1-\theta) f\left(\frac{a+b}{2}\right)+\theta f(b)\right] \\
& \quad-\sum_{k=1}^{[(n-1) / 2]} \frac{[1-(n+1) \theta](b-a)^{2 k+1}}{(2 k+1) ! 2^{2 k}} f^{(2 k)}\left(\frac{a+b}{2}\right) \mid \\
& \quad \leq\left\|f^{(n)}\right\|_{\infty} \times \begin{cases}\frac{\left[1-(n+1) \theta+2 n^{n} \theta^{n+1}\right](b-a)^{n+1}}{(n+1) ! 2^{n}}, & n<1 / \theta, \\
\frac{[(n+1) \theta-1](b-a)^{n+1}}{(n+1) ! 2^{n}}, & n \geq 1 / \theta,\end{cases}
\end{aligned}
$$

where $[(n-1) / 2]$ denotes the integer part of $(n-1) / 2$. 
Proof. Putting $x=\frac{1}{2}(a+b)$ in (2.3) with (2.4), (2.5) and (2.6) readily produces the result (2.36).

REMARK 2.8. If we take $\theta=0$ in (2.36), we get the sharp midpoint type inequality

$$
\begin{aligned}
& \left|\int_{a}^{b} f(x) d x-(b-a) f\left(\frac{a+b}{2}\right)-\sum_{k=1}^{[(n-1) / 2]} \frac{(b-a)^{2 k+1}}{(2 k+1) ! 2^{2 k}} f^{(2 k)}\left(\frac{a+b}{2}\right)\right| \\
& \quad \leq \frac{(b-a)^{n+1}}{(n+1) ! 2^{n}}\left\|f^{(n)}\right\|_{\infty}
\end{aligned}
$$

which appeared in [1] without a proof of sharpness.

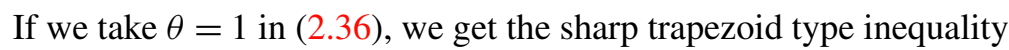

$$
\begin{aligned}
& \left|\int_{a}^{b} f(x) d x-\frac{b-a}{2}[f(a)+f(b)]+\sum_{k=1}^{[(n-1) / 2]} \frac{k(b-a)^{2 k+1}}{(2 k+1) ! 2^{2 k-1}} f^{(2 k)}\left(\frac{a+b}{2}\right)\right| \\
& \quad \leq \frac{n(b-a)^{n+1}}{(n+1) ! 2^{n}}\left\|f^{(n)}\right\|_{\infty} .
\end{aligned}
$$

If we take $\theta=\frac{1}{3}$ in (2.36), we get the sharp Simpson type inequality

$$
\begin{gathered}
\mid \int_{a}^{b} f(x) d x-\frac{b-a}{6}\left[f(a)+4 f\left(\frac{a+b}{2}\right)+f(b)\right] \\
+\sum_{k=1}^{[(n-1) / 2]} \frac{(k-1)(b-a)^{2 k+1}}{3(2 k+1) ! 2^{2 k-1}} f^{(2 k)}\left(\frac{a+b}{2}\right) \mid \\
\leq\left\|f^{(n)}\right\|_{\infty} \times \begin{cases}\frac{5}{36}(b-a)^{2}, & n=1, \\
\frac{1}{81}(b-a)^{2}, & n=2, \\
\frac{(n-2)(b-a)^{n+1}}{3 n+1) ! 2^{n}}, & n \geq 3\end{cases}
\end{gathered}
$$

which appeared in [2] without a proof of sharpness. 
If we take $\theta=\frac{1}{2}$ in (2.36), we get the sharp averaged midpoint-trapezoid type inequality

$$
\begin{aligned}
& \mid \int_{a}^{b} f(x) d x-\frac{b-a}{4}\left[f(a)+2 f\left(\frac{a+b}{2}\right)+f(b)\right] \\
& +\sum_{k=1}^{[(n-1) / 2]} \frac{(2 k-1)(b-a)^{2 k+1}}{(2 k+1) ! 2^{2 k+1}} f^{(2 k)}\left(\frac{a+b}{2}\right) \mid \\
& \quad \leq\left\|f^{(n)}\right\|_{\infty} \times \begin{cases}\frac{1}{8}(b-a)^{2}, & n=1, \\
\frac{(n-1)(b-a)^{n+1}}{(n+1) ! 2^{n+1}}, & n \geq 2 .\end{cases}
\end{aligned}
$$

\section{References}

[1] P. Cerone, S. S. Dragomir and J. Roumeliotis, 'Some Ostrowski type inequalities for $n$-time differentiable mappings and applications', Demonstratio Math. 32(4) (1999), 697-712.

[2] Z. Liu, 'An inequality of Simpson type', Proc R. Soc. Lond. Ser. A Math. Phys. Eng. Sci. 461 (2005), $2155-2158$.

[3] Z. Liu, 'Some Ostrowski type inequalities', Math. Comput. Modelling 48 (2008), 949-960.

[4] C. E. M. Pearce, J. Pečarić, N. Ujević and S. Varošanec, 'Generalizations of some inequalities of Ostrowski-Grüss type', Math. Inequal. Appl. 3 (2000), 25-34.

ZHENG LIU, Institute of Applied Mathematics, School of Science,

University of Science and Technology Liaoning,

Anshan 114051, Liaoning, PR China

e-mail: lewzheng@163.net 